\title{
Intussusception- A Case Report
}

Gurubacharya SM', Gurubacharya RL ${ }^{2}$

${ }^{1}$ Dr. Simmi M Gurubacharya, MD Paediatrics, Lecturer, ${ }^{2}$ Dr. Rajesh L Gurubacharya, MD Paediatrics, Assistant Professor, Department of Pediatrics, College of Medical Sciences, Kathmandu University.

Address for Correspondence: Dr. Rajesh Lal Gurubacharya

E-mail: rajesh_pul@hotmail.com

\begin{abstract}
Intussusception, the invagination of a portion of the intestine into itself, is one of the emergencies in infancy and childhood. The etiology may be idiopathic or secondary to some pathology within the wall of the bowel. Most cases (90\%) are idiopathic ${ }^{1,2}$ with no identifiable lesion acting as the lead point or pathological apex ${ }^{3}$ of the intussusceptum Children may present at any age but this occurs most commonly in the first year. The mode of presentation may vary depending upon the time of presentation. A case of intussusception confirmed with the help of an abdominal ultrasound in 10 month old infant who presented with only persistent vomiting but in the absence of classic features is reported with brief review of literature.
\end{abstract}

Key words: vomiting, USG, classic triad

\section{Introduction}

Intussusception (IS), the most common cause of acute bowel obstruction in infants and young children which commonly occurs at the terminal ileum (i.e.ileocolic). The telescoping proximal portion of bowel (i.e. intussusceptum) invaginates into the adjacent distal bowel (i.e. intussuscipiens). Most patients recover if treated within 24 hours. If left untreated, this condition is uniformly fatal in 2-5 days. Mortality with treatment is $1-3 \%$ Recurrence is observed in $3-11 \%$ of cases. Most recurrences involve intussusceptions that were reduced with contrast enema. Overall, the maleto-female ratio is approximately $3: 1$. Intussusception is most common in infants aged 3-12 months, with an average age of 9 months $^{4}$. Intussusception occurrence is rare in persons younger than 3 months, and it becomes less common in persons older than 36 months. The classic triad of colicky abdominal pain, vomiting, red currant jelly stools or abdominal mass occurs in $12.5 \%-46 \%$ of cases ${ }^{4}$.

Ultrasonography in Pediatrics; an accurate, safe and valuable clinical tool is being increasingly used as the primary investigation for the diagnosis of IS and to guide air or hydrostatic enema reduction ${ }^{4,5}$. The use of ultrasonography for a patient with suspected IS prevents unnecessary radiological or surgical procedures being performed, and reduces radiation exposure while maintaining a high level of diagnostic accuracy ${ }^{5}$. When small-bowel intussusception is detected in infants and children undergoing abdominal sonography, intussusception length greater than $3.5 \mathrm{~cm}$ is a strong independent predictor of the need for surgical intervention ${ }^{6}$.

\section{Case Report}

A 10-month-old female infant was brought to the OPD with complaints of persistent vomiting, non-bilious, nonprojectile in nature for two days for which the child was given anti-emetics from outside. According to the mother, there was no history of fever, loose stools mixed with blood and mucus, coryza, cough, any urinary problems, nor convulsions or rashes over the body. However, the mother told that the child was crying excessively, intermittently and was noticed to be in distress due to vomiting. The patient was born at hospital; birth weight was normal. Her immunisation was complete according to EPI (national) schedule. Her feeding history was also normal. The developmental milestones were appropriate for age.

On physical examination, child was of average built. Her height, weight and head circumference were within normal limits. Pulse rate, respiratory rate, temperature and BP were normal. She was anicteric. There were no features of dehydration. Her hernial orifices were normal. Rest of the general examination was also normal. Systemic examination revealed no abnormalities. All routine investigations including SGOT, SGPT were normal. Finally USG of the whole abdomen was advised because of the major symptom i.e. the persistent vomiting was present without pointer to rule out or confirm any surgical condition. However, USG confirmed (Fig.1) the diagnosis, which was a suspected intussusception, the most common cause of acute intestinal obstruction in infants.

Fig.1: USG Showing Loops with the Loops of Bowel

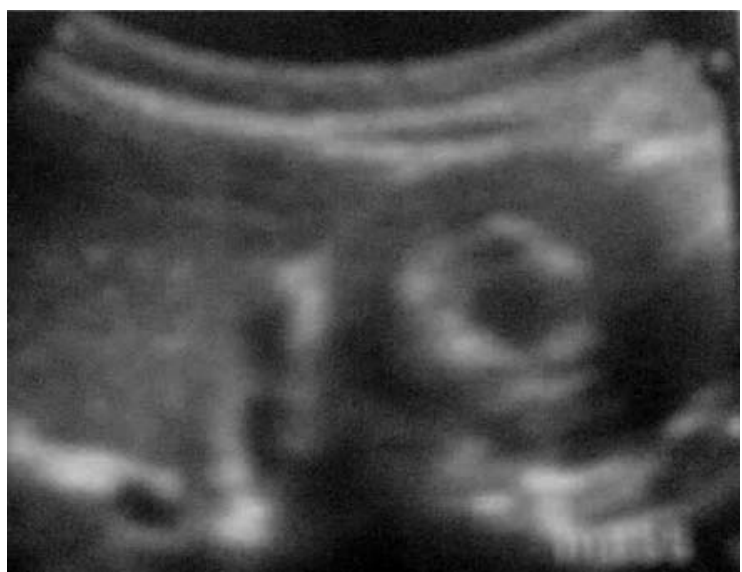




\section{Discussion}

Intussusceptions commonly occur below one year ${ }^{7}$, a finding confirmed in our case. Intussusception is said to occur in well-nourished infants ${ }^{8}$, though also seen in many malnourished children. The patients usually present with symptoms of pain, vomiting, blood in the rectal discharge and a palpable mass but not occurred in our case except vomiting. Early on, the abdomen is flat or scaphoid, as time passes distension and intestinal loops may obscure the mass.

Intussusceptions, can be of various types like the ileoileal, ileo-caecal, ileo-colic, ileocaeco-colic, jejuno-ileal, colo- colic of which the ileo-colic was the most common and only $2-8 \%{ }^{7,8,9}$ of intussusceptions have a recognizable lesion acting as the lead point. Various lesions have been cited like Polyps, Meckel's diverticulum, Hypertrophied Lymphoid Patch, Human Reovirus like agent a cause of gastroenteritis in children $^{8}$, Enteric Cysts, Ectopic Pancreatic Tissue, and even Henoch-Schonlein purpura $^{8}$. It can occur after abdominal operative manipulations like Eke resection of coarctation of aorta, resection of Wilms tumour, biopsy for neuroblastoma.

Due to the pathogenesis of this disease, the mesentery of the invaginated bowel is compressed between the layers of the intussusceptum, hence the longer it remains unreduced the higher the chances of gangrene setting in and lesser chances of reduction (of the intussusception). In 1871, Hutchinson successful operated upon an infant with intussusception8. Shortly afterward Hirschsprung ${ }^{10}$ reported a series of children whose intussusception was reduced by retrograde hydrostatic pressure. Since that time the mode of therapy has become controversial. More and more institutions are shifting to barium enema reduction as primary treatment in most of their patients.

To achieve success with this method, certain principles must be vigorously observed ${ }^{8}$. The pre-requisites before attempting a barium enema are that duration of symptoms should be less than 2 days as not happened in our case, there should not be signs of intestinal obstruction on abdominal radiography, and there should be no signs of localised or generalised peritonitis. The barium enema reduction is confirmed if there is free flow of barium into the terminal ileum, expulsion of faeces and flatus with barium, disappearance of the mass, and clinically child becomes comfortable. Reduction attempts are abandoned ${ }^{9}$ if the intussusception does not move for more than 10 minutes; there is partial reduction, a poor ileal reflux, a persistent mass in the ileum, or leak of barium suggesting colonic perforation ${ }^{11}$. The benefits of barium enema reduction procedure are complete reduction without surgery, no anaesthesia is required, no morbidity, early ambulation, less stay in the hospital, no scar. The disadvantages are its uselessness in ileo ileal intussusception; recurrence rate is higher (11\%) than after operative reduction $(3 \%)^{11}$, specific recognizable lead points can be missed $(2-8 \%)$, in unsuccessful cases a dangerous delay, colonic perforation ${ }^{11}$, and the possibility of reducing nonviable bowel.

The standard treatment of intussusception used to be operative reduction by milking back of the intussusception by progressive compression of the bowel just distal to it as done in our case. If reduction is not possible, or gangrene has set in, resection of the affected bowel has to be done, followed by either Mickulicz procedure or a primary end-toend anastomosis which did not happen in our child. A newer mode of therapy introduced by the Chinese is the concept of air pressure enema reduction of intussusception ${ }^{12,13}$.

\section{Conclusion}

The 'classic' picture of intussusception (vomiting, abdominal pain, bloody/red currant jelly stool, or abdominal mass) might not be frequently present in children with intussusception. Reliance on 'classic' features alone might delay diagnosis. Median time to confirmation of diagnosis is usually 19 hours from onset of symptoms ${ }^{4}$. Delayed diagnosis is associated with poorer patient outcomes. Thus in the light of above mentioned case, child with persistent vomiting without pointer may need to be differentiated from common surgical condition particularly in an infant to prevent fatality. Also the importance of USG is stressed, which is a simple and useful method of making the diagnosis of intussusception.

\section{Acknowledgement}

The author would like to acknowledge the parents who kindly consented for publication. The authors are thankful to the Director and the hospital management for granting permission to publish the case report.

\section{References}

1. Kim YS, Rhu JH. Intussusception In Infancy and Childhood: Analysis of 385 Cases. Int Surg 1989; 74:114-118.

2. AI-Salem AH, Habash 8M. Ileoileal Intussusception: A Report of Four Cases. Ann Saudi Med 2000; 20:310312.

3. Stringer MD, Pablot SM, Brereton RJ. Pediatric Intussusception. Br J Surg 1992; 79: 867-876

4. Blanch AJ, Perel SB, Acworth JP. Paediatric Intussusception: Epidemiology And Outcome. Emerg Med Australas 2007; 19(1):45-50.

5. Justice FA, de Campo M, Liem NT, Son TN, Ninh TP, Bines JE. Accuracy of Ultrasonography For The Diagnosis Of Intussusception In Infants In Vietnam. Pediatr Radiol. 2007; 37(2):195-199.

6. Munden MM, Bruzzi JF, Coley BD, Munden RF. Sonography Of Pediatric Small-Bowel Intussusception: Differentiating Surgical From Nonsurgical Cases: AJR Am J Roentgenol. 2007; 188(1):275-9.

7. Dennison WH, Shaker M. Intussusception In Infancy And Childhood. Brit J Surg 1970; 57:679-684.

8. Ravitch MR. Intussusception. In: "Paediatric Surgery", 4th Ed.Welch KJ, Randolph JG, Ravitch MM, O’Neill JA, Rowe MI, editors. Chicago: Year Book Medical Publishers, Inc; Chicago, 1986, pp 868-881.

9. Rosenkrantz JG, Cox JA, Silverman FN, Martin LW. Intussusception In 1970s: Indication For Operation. J Paed Surg 1977; 12:367-373. 
10. Hirschsprung H. Tilfaelde Of Subakut TanninVagination. Hospitalstid 1876; 3:321-327.

11. Ein SH, Mercer S, Humphrey A, Mac-donald P. Colon Perforation During Attempted Barium Enema Reduction. J Paed Surg 1981; 16:313-315.
12. Guo JZ, Ma XX, Zhou QH. Results of Air Pressure Enema Reduction Of Intussusception 6396 Cases: 13 Years. J Paed Surg 1986; 21:1201-1203.

13. Jinzine Z, Yenxia W, Linchi W. Rectal Inflation Reduction Of Intussusception In Infants. J Paed Surg 1986; 21:12-13. 